\title{
The best of SBP-BRiMS
}

\author{
Kathleen M. Carley ${ }^{1}$
}

Published online: 16 May 2020

๑) Springer Science+Business Media, LLC, part of Springer Nature 2020

Computational social-science brings together computer science, data science, statistics, and the social sciences to address complex real world issues. This conference celebrates this merger exploring advances in socio-cultural, behavioral, information and network analysis and modeling. Interdisciplinary research merging distinct fields are presented as are transdisciplinary research seeking to create and test new theories. A large number of topics are considered including, but by no means limited to: group formation, interaction, and evolution, collective action, public opinion representation, and governance, social conventions, social contexts and influence processes, intelligent agents and adversarial modeling, performance prediction, assessment, \& skill monitoring/tracking, social media assessment, data mining and analytics given digital trace data, information and belief diffusion, high dimensional network analytics, social impacts of disease, and team science.

This special issue represents the best of this conference. It includes: papers based on a keynote presentation, winners of the best papers (student, general, late breaking), and papers about the response to a challenge event by the winners. These papers all have in common the fact that they go beyond a single disciplinary perspective, employ relatively novel techniques, and address challenging issues.

Please join the conference in 2020 when additional issues related to validation, simulation, and covid-19 will also be included as part of special panels.

Publisher's Note Springer Nature remains neutral with regard to jurisdictional claims in published maps and institutional affiliations.

Kathleen M. Carley

kathleen.carley@cs.cmu.edu

1 School of Computer Science, Carnegie Mellon University, Pittsburgh, PA, USA 